E.L.U.A. 3, 1985-1986, págs. 193-208

\title{
ANÁLISIS DE LAS LEXÍAS COMPLEJAS EN DOCUMENTOS MEDIEVALES MURCIANOS*
}

\author{
PILAR DIEZ DE REVENGA \\ (Universidad de Murcia)
}

En el presente trabajo tratamos de abordar el análisis de la estructura de unos documentos medievales escritos en el Reino de Murcia en el siglo XIII.

Del conjunto de documentos transcritos por el Dr. Torres Fontes y publicados en la "Colección de Documentos para la Historia del Reino de Murcia" hemos utilizado algunos de los recogidos en el volumen II ', que agrupa documentos particulares. De ellos hemos elegido un total de doce que, atendiendo a su contenido, podriamos clasificar desde el punto de vista jurídico como sigue:

1) Donaciones: Se trata en estos casos de una donación doble (doc. II) y dos donaciones simples (docs. XVIII y LI) ${ }^{2}$. En estas cartas no es imprescindible que se exprese el motivo de tal acto, aunque en ocasiones si se hace.

* Un esbozo del presente artículo fue presentado como comunicación del mismo título en el XIil Simposium de la Sociedad Española de Lingüística, Barcelona, 1983. Más tarde ha sido ampliado considerablemente.

1 Torres Fontes (1969). Conservamos la numeración de los documentos utilizada en esta edición y la citamos entre paréntesis al referirnos a cada documento estudiado.

2 Lalinde Abadía (1970: 685-686). Empleamos la abreviatura D cuando nos referimos a estas cartas. 
2) Concesión de bienes como ejecución de unas capitulaciones matrimoniales (Doc. LII). ${ }^{3}$

3) Censos: recogemos en este apartado dos cartas de censo consignativo (docs. XXI y LVIII) y una de censo enfitéutico (doc. XXXVII) ${ }^{4}$.

4) Arrendamiento: incluimos una carta de arrendamiento de tierras, animales, etc. (doc. LVI) y otra que, si bien no es de arrendamiento propiamente dicho, trata de pago de rentas (doc. LV) ${ }^{5}$.

5) Compensación: este documento representa el pago o cumplimiento de la deuda como efecto de la obligación (doc. LIV). Este apartado podría englobar también una carta de composición (doc. XLVI) .

6 Venta: Es un documento que representa el arquetipo de contrato oneroso y bilateral (doc. LVII) (Lalinde Abadia, 1970: 686 y ss.).

Estas transacciones se llevan a cabo bien entre particulares, bien entre Ordenes Militares, o la iglesia, y particulares y están redactadas entre 1243 y 1283, fechas clave para la reconquista y repoblación del Reino de Murcia. El hecho de que hayamos centrado nuestra atención en los documentos particulares se debe a la diversa condición de los escribanos y al deseo de no interferir este trabajo con las estructuras propias de los asuntos de la Corona.

Para la elaboración del trabajo hemos considerado cada documento como un texto independiente de los demás y con entidad propia, si bien no hemos tenido en cuenta, en ocasiones, el escriba que los firma, ya que algunos son anónimos y en otros sólo consta el notario.

La división siguiente ha sido realizada siguiendo los esquemas que propone la diplomática según la cual los documentos medievales se componen de los apartados siguientes: exordium, notificatio, narratio, dispositio, sanctio y corroboratio ${ }^{7}$. En ellos puede faltar, y falta generalmente, el exordium. De hecho en los documentos estudiados únicamente presenta exordium uno (doc. XXI). La notificatio, en la que se encuadra el complejo emisor, se presenta en todos: incluso el n. $^{\circ} \mid 1$ nos ofrece doble notificatio como expresión de una cesión mutua entre el maestre D. Pelay Perez y el comendador D. Gil Gómez.

Asimismo la narratio, de diversa extensión según el asunto tratado,

3 Lalinde Abadía (1970: 621 y ss.). Aunque el documento LIl no es una donación, utilizamos también la abreviatura $\mathrm{D}$ cuando tratamos de él.

4 Lalinde Abadia (1970: 622 y ss.). La abreviatura en este caso es C.

5 Lalinde Abadia (1970: 691 y ss.). Abreviatura A.

6 Lalinde Abadia (1970: 673). Compensación (CPE) y Composición (CPO).

7 Metzeltin (1982: 149-150); Pascual Martínez (1982), donde se presenta una clasificación similar de las cartas de donación: Protocolo inicial (Notificación, intitulación), Texto (Exposición, Disposición y Cláusulas) y Protocolo final (Data y suscripciones); Ser Quijano (1981: 9-33). 
consta en todos ellos. Este apartado incluye el objeto de la donación y su descripción, situación y límites.

La dispositio, en la que se indican las condiciones del disfrute y plazo de pagos de la venta o arrendamiento llevado a cabo, se desarrolla en nueve documentos y falta en tres, referidos éstos a donaciones y cesiones.

La sanctio que, como su nombre indica, hace explícitas las sanciones que ocasionaría el incumplimiento de los pactos, aparece en seis documentos y falta en otros seis, que atañen a donaciones.

Por tanto es frecuente hallar dispositio y sanctio en el mismo documento; en menor proporción encontramos cartas que ofrezcan solamente uno de los dos apartados: o dispositio o sanctio (vid. cuadro 1).

\section{CUADRO I}

\begin{tabular}{|c|c|c|c|c|c|c|c|c|c|c|c|c|}
\hline & 11 & XVIII & $\mathbf{x} \mathbf{x I}$ & XXXVII & XLVI & LI & LII & LIV & LV & LVI & LVII & LVIII \\
\hline Exordium & - & - & + & - & - & - & - & - & - & - & - & - \\
\hline Notificatio & & + & + & + & + & + & + & + & + & + & + & + \\
\hline Narratio & & + & + & + & + & + & + & + & + & + & + & + \\
\hline Dispositio & + & + & + & + & + & - & + & - & - & + & + & + \\
\hline Sanctio & - & - & - & + & - & - & + & + & - & + & + & + \\
\hline Corroboratio & + & + & + & + & + & + & + & + & + & + & + & + \\
\hline
\end{tabular}

+ Presenta dicho apartado.

- No presenta dicho apartado.

Como vemos en el cuadro l, tan sólo dos documentos coinciden en carecer de dispositio y sanctio.

La corroboratio en donde se establece la veracidad y firmeza del hecho descrito, así como la presencia de testigos, la legalización del documento, el escriba o notario y la fecha, al igual que la narratio y la notificatio, aparece en todos.

Cada una de las partes enumeradas presenta una estructura muy si- 
milar en cada carta que se corresponde con la estructura lingüística en donde cada documento representa una PROPOSICION, cuyos elementos -a grandes rasgos _ serían los siguientes (Metzeltin, 1982: 149): un primer segmento en donde la característica general es la presencia de sujetos que donan, ceden o arriendan una propiedad y que corresponderia a la narratio de la clasificación anterior.

El segundo segmento constituye la indicación del futuro estatuto de la propiedad y equivaldria a la dispositio.

En el tercero se harian explícitas las sanciones por incumplimiento del contrato y sería igual a la sanctio.

A continuación, se expresarian las garantías para el beneficiario, la enumeración de testigos, la confirmación del acto y, por último, la fecha y firma, que encontraría su paralelo en la corroboratio.

Estos apartados, con una organización muy similar en cada texto, se relatan mediante fórmulas emitidas de un modo casi idéntico en su desarrollo, aunque ofrecen variantes que amplían el núcleo de la proposición.

Estas fórmulas, constituidas por el uso en lexías complejas (Pottier, 1974 :266) de índole jurídica, en opinión de Roudil (1981; 1974), suelen presentarse agrupadas en cada apartado de los mencionados anteriormente; pero en ocasiones, cambian de lugar, apareciendo en el apartado inicial y reiterándose a lo largo del texto con lo cual vamos observando, paso a paso, la estructura repetitiva de los documentos medievales.

No pretendemos en este momento que el análisis de las lexias sea exhaustivo, sino sólo hacer mención de aquellas que aparecen con mayor frecuencia, así como de sus variantes. En él no consideraremos alterada la lexía cuando cambia el número: singular o plural según sea el emisor único o múltiple. El cambio de número no obedece a otras causas más que a éstas. En cuanto a la variación del tiempo verbal o su sustitución por una perifrasis en principio sí las consideramos como tales.

En la notificatio encontramos los documentos elegidos divididos en dos bloques equivalentes, sin que el asunto que refleja el texto (venta, donación, etc.), influya en la fórmula de llamamiento. El primero se expresa con la lexía compleja tipo CONOCIDA COSA SEA A CUANTOS ESTA CARTA VIEREN, que si en principio está constituida por Part. $+\mathrm{N}+$ $+\mathrm{V}+$ Pron. + Adj. $+\mathrm{N}+\mathrm{V}$, puede ampliarse y hasta variar algunos de sus elementos. En los seis casos registrados no hay ninguno idéntico, aunque su base sea la misma. Las variantes observadas, y en orden creciente, son las siguientes: 
CONOÇUDA COSA SEA A quantos ESTA CARTA VEYEREN como Orrigo Porçell (doc. XXXVII, C)

CONOSÇUDA COSA SEA A quantos ESTA CARTA UIEREN como nos por la gracia de Dios (doc. LVI, A)

CONOCIDA COSA SEA A los que an por uenir quomo nos por la gracia de Dios (doc. II, D)

CONOCIDA COSA SEA A todos que ESTA CARTA VIEREN como yo sana et alegre et en toda mi memoria ... (doc. XVIII, D)

CUNUÇUDA COSA A todos Ios omnes que ESTA CARTA VIEREN ET OYEREN como Yo (doc. XXI, C)

CONNOÇUDA COSA SEA SEA A quantos ESTA CARTA VIEREN ET OYEREN commo nos (doc. XLVI, CPO.)

El segundo bloque de lexias complejas observadas en la notificatio está compuesto por SEPAN QUANTOS ESTA CARTA VIEREN; se amplía como la anterior, pero no ofrece más variantes en su expresión. Si consideramos como base $\mathrm{V}+$ Pron. $+\mathrm{Adj}$. $+\mathrm{N}+\mathrm{V}$, vemos que se repite en los seis casos idéntica, para desarrollarse en algunos con otros complementos, que a su vez pueden constituirse en lexías complejas. Las variantes son las siguientes:

SEPAN QUANTOS ESTA CARTA UIEREN COMMO nos (doc. LI, D) SEPAN QUANTOS ESTA CARTA VIEREN COMMO nos (doc. LIV, CPE) SEPAN QUANTOS ESTA CARTA VIEREN COMMO nos por la gracia de Dios (doc. LV, A)

SEPAN QUANTOS ESTA CARTA UJEREN COMMO yo de grado et buen corasson (doc. LVII, V)

SEPAN QUANTOS ESTA CARTA UJEREN et oyeren COMMO nos (doc. LVIII, C.)

SEPAN QUANTOS ESTA CARTA UIEREN et oyeren COMMO nos (doc. LII, D)

El encabezamiento de la narratio presenta varios tipos de lexías según el asunto de la carta, que suelen expresarse mediante pares de verbos. Como antes indicábamos, no tenemos en cuenta la persona en que se conjuga el verbo, ya que esto es debido a que el emisor sea una o más personas.

En primer lugar, hallamos aquellas lexías complejas constituidas sobre la base de verbos que aparecen aislados en otras ocasiones y que funcionarían como lexías múltiples combinatorias. En ellas se asocia DAR + OTORGAR, cuya base estaría formada por $V+V+$ Pron.

DAMOS et OTORGAMOS a uos (doc. II, D)

DO et OTORGO (doc. II, D)

OTORGO et DO de buena uoluntat et de buen coraçon (doc. XVIII, D) OTORGAMOS que DIEMOS (doc. LIV, CPE) 
OTORGAMOS que nos DIESTES (doc. LVI, A)

DO et OTORGO (varios documentos)

y en la que se altera el orden: unas veces aparece DAR como primera parte de esta lexía compleja y, otras, OTORGAR. El primer lugar está ocupado por DAR en las cartas de donación generalmente y OTORGAR en las de arrendamiento y compensación.

Del mismo modo DAR se combina por una parte con ESTABLECER en los documentos de arrendamiento y se amplía sobre la base $V+V$ con la adición de pronombres únicamente:

DO et ESTABLESCO (doc. XXI, C)

uos la DO et uos la ESTABLESCO (doc. XXXVII, C)

DO et ESTABLESCO (doc. LVIII, C)

$y$, por otra, con ENTREGAR, que aparece una sola vez en los documentos que nos ocupan, en una donación:

DIEMOS et ge lo ENTREGAMOS

Hemos visto que DAR se combinaba con OTORGAR y otros verbos. Ahora vamos a analizar las posibilidades que ofrece OTORGAR, asociada, siempre en orden creciente, a VENDER con una sola aparición en una escritura de venta:

VENDO et OTORGO vendida buena pora siempre jamas valedera (doc. LVII, V)

Asimismo, OTORGAR aparece junto a CONOCER con una base $V+V$, en donde el segundo verbo está representado dos veces por una perífrasis:

OTORGO et CONOSCO por el poder... (doc. XXXVII, C)

OTORGAMOS et VENIMOS CUNUSÇUDO (doc. LV, A)

Et OTORGO et UENGO de CONOÇUDO (doc. LVII, V)

Por último, OTORGAR se combina con PROMETER y, como en los casos anteriores, la lexía compleja se establece sobre una base de $\mathrm{V}+\mathrm{V}$, en donde los otros elementos registrados se limitan a la categoría de pronombres:

OTORGAMOS et PROMETEMOS (doc. XLVI, CPO)

Et OTORGAMOS uos et PROMETEMOS uos (doc. LVI, A)

Otrossi uos OTORGAMOS et uos PROMETEMOS bien et cumplidament (doc. LVI, A)

Et OTORGO et PROMETO (doc. LVII, V)

Et OTORGO et PROMETO a uos (doc. LVIII, C)

También encontramos ARRENDAR, cuya base sería Pron. $+\mathrm{V}+$ Compl., en donde el orden sólo se altera una vez:

ET OTROSSI UOS ARRENDAMOS EN ESTOS ARRENDAMIENTOS (doc. LVI, A) 
ET OTROSSI UOS ARRENDAMOS EN ESTOS ARRENDAMIENTOS (doc. LVI, A)

ET OTROSSI UOS ARRENDAMOS EN ESTOS ARRENDAMIENTOS (doc. LVI, A)

ET OTROSSI UOS ARRENDAMOS EN ESTOS ARRENDAMIENTOS (doc. LVI, A)

DE TODOS ESTOS ARRENDAMIENTOS sobredichos UOS ARRENDAMOS (doc. LVI, A)

Hemos de señalar con respecto a estas lexías que si bien aparecen generalmente en el apartado correspondiente a la narratio con índice superior de frecuencia, también las encontramos en otros apartados como la dispositio y corroboratio y, con menor incidencia, en la sanctio. Esto demuestra una vez más que las lexías que forman la macroestructura de las cartas privadas medievales son esencialmente repetitivas.

Otra de las lexías complejas inventariadas en la narratio la constituye la formada por dos participios sustantivados

CON ENTRADAS ET CON SALLIDAS (varios docs.)

La lexía aparece inalterada en todas sus apariciones, aunque en algunas de ellas se ve incrementada con adiciones que, por sí mismas, se podrian considerar también como lexías complejas.

Una sola vez la hallamos en la dispositio, en lugar de en la narratio; esto se debe al diferente orden de enumeración de las cualidades del objeto del que se trata, independientemente de qué tipo sea la transacción realizada:

CON ENTRADAS ET CON SALLIDAS (doc. LVIII, C)

CON ENTRADAS ET CON SALLIDAS et con todas sus pertenencias (doc. LVII, V)

CON ENTRADAS ET CON SALLIDAS et con todas sus pertenencias (doc. XXXVII, C)

CON ENTRADAS ET CON SALLIDAS et con meioramientos et con todos sus derechos (doc. XXI, C)

CON ENTRADAS ET CON SALLIDAS et con aldeas, ... et con todos los derechos (doc. II, D)

CON ENTRADAS ET CON SALLIDAS et con ... et con todas sus pertenencias et derechos (doc. XVIII, D)

En la narratio, y como en casos analizados en otros apartados, la siguiente lexía compleja observada es la constituida por dos adjetivos como base: LIBRE ET QUITO, que se amplía adjuntando un verbo. Otras veces se desarrolla, además, sumando otra lexía compleja. Aparece en todo tipo de actos, excepto en casos de arrendamiento, lo cual es lógico: 
LIBRA ET QUITA (doc. LVII, V)

Que finque LIURE ET QUITE (doc. II, D)

Que lo ayades LIBRE ET QUITO por jure de heredat pora siempre (doc. LIV, CPE)

Que lo ayades LIBRE ET QUITO por jure de heredat pora siempre (doc. LI, D)

Que lo aya LIBRE ET QUITO por juro de heredat pora siempre (Doc. LII, D)

o bien, esta otra, compuesta sobre una base de $\mathrm{N}+\mathrm{N}$ :

... todo quanto ouiessen, MUEBLE ET RAYZ (doc. LII, D)

... mi et todos mis bienes MUEBLES ET RAYZ (doc. LVIII, C)

Cuando se trata de fijar plazos de pago de arrendamientos se emplean una serie de fórmulas que ratifican estos vencimientos y son fechas que aún perduran, al menos, en la huerta de Murcia. Está constituida esta lexía por Prep. + Pron. + N. :

POR LA SAN JUAN (doc. LVI, A)

desde esta SAN JUAN (doc. LVI, A)

de ESTE SAN JUAN que pasó (doc. LVI, A)

Por LA SAN JOHAN de junio (doc. LVIII, C)

Fasta LA otra SAN JOHAN (doc. LV, A)

En el año que se començo por LA SAN JOHAN (doc. LV, A)

POR LA festa de SAN JOHAN de junio (doc. XLVI, CPO)

POR LA fiesta de SAN JOHAN del mes de junio (doc. XXXVII, C)

POR LA fiesta de SAN ANDRES (doc. XXI, C)

POR LA NAUIDAD (doc. LVIII, C)

Estas lexias suelen ir acompañadas de otras tal como:

CADA ANNO (doc. XLVI, CPO)

POR CADA ANNO (doc. XXI, C)

POR CADA ANYO ... DE ANYO EN ANYO (doc. XXXVII, C) y expresadas en la dispositio.

La corroboración del acto que se narra, ya sea donación, compra o arrendamiento, se hace explícita por medio de varias lexías, o por medio de una lexía múltiple. En primer lugar hallamos:

SEA FIRME et pora çiempre jamas (doc. XXI, C)

SEA FIRME et estable (doc. XLVI, CPO)

Por otra parte encontramos:

mas creudo et NON VENGA EN DUBDA (doc. XXXVII, C)

NON UENGA EN DUBDA (doc. LI, D)

NON UENGA EN DUBDA (doc. LIV, CPE)

Esta última aparece combinada con la anterior y así registramos tres casos:

SEA mas FIRME ET NON UENGA EN DUBDA (doc. LII, D) 
SEA FIRME ET NON UENGA EN DUBDA (doc. LV, A) SEA FIRME ET NON UENGA EN DUBDA (doc. LVI, A)

Todas ellas van precedidas generalmente por Et porque este hecho.

Otro modo de expresión que, en los documentos elegidos, se presenta una sola vez es la lexía MAS FORTO ET MAS FIRMADO.

Otra lexía compleja de frecuente aparición es aquella que determina alguna caracteristica del documento legalizado, y que se repite idéntica, sobre una base de Part. $+\mathrm{N}$. :

PARTIDAS POR A.B.C. (doc. LVI, A)

PARTIDAS POR A.B.C. (doc. XLVI, CPO)

PER A.B.C. PARTIDAS (doc. II, D)

PARTIDAS POR letras (doc. XXI, C)

En el marco de la corroboratio se repiten otras lexías que ponen de manifiesto la legalidad del pacto. Entre ellas señalamos las siguientes:

SEELLADA CON NOSTRO SELLO

cuya base formada por Part. + Prep. + Adj. + $\mathrm{N}$ se mantiene, aunque altere el orden en una ocasión, y se amplía con algún adjetivo referente al sello y a su clase, así como a la categoría de la escribanía

SELLADA CON NOSTRO SELLO (doc. LV, A)

SELLADAS CON NOSOTROS SELLOS (doc. LVI, A)

Con IOS NUESTROS SIELOS SEELADAS (doc. II, D)

SEELLADO CON NOSTROS SEELLOS colgados (doc. LIV, CPE)

SELLADA CON NUESTROS SEELLOS colgados (doc. LI, D)

Mande SEELLAR esta carta con MIO SELLO colgado (doc. XXXVII, O)

SEELLADA CON NOSOTROS SEELLOS pendentes (doc. LII, D)

SEELLADAS CON el NOSTRO SELLO ... ET CON el uostro (doc. XLVI, CPO)

La lexía compleja que ratifica la autoría del escribano se desarrolla sobre una base compuesta por Part. + Orac. de relativo:

SIGNUM QUI HOC SCRIPSIT (doc. XVIII, D)

SENNAL DE MI QUI ESTA CARTA ESCREUI (doc. LVII, V)

SENYAL DE MI QUI ESTA CARTA ESCREUI (doc. LVIII, C)

Otro modo de acreditar el documento es indicando no el escribano, sino el notario. La lexía utilizada en este caso es similar a la anterior. Unicamente sustituye los elementos de la oración de relativo:

SIGNUM QUE ESTA CARTA FIZ ESCRIUIR et enserte por mandamiento (doc. XXI, C)

Esta presenta una variante: LA MANDE FAZER por mandado del maestre (doc. LV, A)

Los restantes documentos son anónimos y, lógicamente, carecen de este tipo de lexías. 
La enumeración de los testigos la encontramos expresada con lexías similares:

SUM TESTIS (doc. XVIII, D)

TESTIGUOS desto SON (doc. XXI, C)

Et estos SON TESTIGOS (doc. LII, D)

TESTIGOS FUERON ROGADOS (doc. LVIII, C)

TESTIGOS FUERON desto ROGADOS (doc. LVII, V)

en donde la base $V+V$ se amplía con la adición de pronombres. Sólo una vez hallamos expresada la presencia de testigos con la lexía ET A ESTA CUENTA SE ACERCARON (doc. LV, A). En los restantes documentos analizados no constan los testigos.

La data de la carta la encontramos representada por la lexía compleja FECHA EN más el nombre de la ciudad, cuya base Part. + Prep. + $\mathrm{N}$ se amplia sumando otro nombre:

ACTUM FUIT (doc. XVIII, D)

FECHA EN (ciudad) (doc. II, D)

FECHA EN (ciudad) (doc. XLVI, CPO)

FECHA EN (ciudad) (doc. LI, D)

FECHA la carta EN (ciudad) (doc. XXI, C)

FECHA la carta EN (ciudad) (doc. XXXVII, C)

FECHA la carta EN (ciudad) (doc. LII, D)

FECHA la carta EN (ciudad) (doc. LV, A)

FECHA la carta EN (ciudad) (doc. LVII, V)

FECHA la carta EN (ciudad) (doc. LVIII, C)

FECHA la aluara EN (ciudad) (doc. LIV, CPE)

Las quales fueron FECHAS EN (ciudad) (doc. LVI, A)

Por último, otro aspecto de la data lo hallamos representado por la lexía compleja que incluye el día de la semana y su correspondiente del mes:

HOC JOUES DIES APRILIS (doc. XVIII, D)

JUEUES... DIAS DE SETEMBRE (doc. XLVI, CPO)

MIERCOLES ...DIAS DE JUNIO (doc. LI, D)

MIERCOLES... DIAS DE JULIO (doc. LII, D)

DOMINGO ... DIAS DE SETIEMBRE (doc. LIV, CPE)

VYERNES ... DIAS DABRIL (doc. LVIII, C)

DIA SABADO ... DIAS DE ABRILL (doc. XXXVII, C)

que se amplía con:

MARTES ... DIAS andados DE FEBRERO (doc. LVII, V)

Die LLUNES ... DIES andados del mes DE MARZO (doc. XXI, C)

Si el día mencionado es el primero o el último del mes se limita a expresarlo de la siguiente forma:

PRIMERO DIA DE AGOSTO (doc. LV, A) 
PRIMER DIA DE AGOSTO (doc. LVI, A)

POSTRIMERO DIA DE ... (doc. II, D)

todas ellas van acompañadas de:

En ERA DE (varios docs.)

ERA DE (varios docs.)

En la ERA... prima (doc. II, D)

o, solamente, un caso: ANNO DOMINI (doc. XVIII, D)

Como consecuencia de todo lo expuesto más arriba, llegamos a las conclusiones siguientes:

1) Los documentos medievales particulares ofrecen un desarrollo de su macroestructura bastante uniforme. Los apartados apuntados anteriormente son iguales y comunes a todos, si bien puede no estar representado alguno de ellos como reflejo del asunto tratado.

2) Dichos apartados se redactan con predominio de un tiempo o un modo verbal determinado en cada uno de éstos (Roudil, 1980). Mientras que la notificatio se emite en subjuntivo, la narratio viene expresada en indicativo como indice de la realización de la transacción expuesta. La dispositio suele redactarse en subjuntivo, pero la sanctio varia. En la corroboratio predomina el participio de pasado, que alterna con el indefinido como expresión de una acción concluida.

3) Las lexías enumeradas hallan su antecedente y, en ocasiones, su correlato en documentos altomedievales escritos en latín. Entre ellas señalamos las siguientes ${ }^{8}$ :

CONOCIDA COSA SEA A QUANTOS ESTA CARTA VIEREN (y variantes):

Notum fieri cupimus omnibus nostris fidelibus cunctisque ... viris, presentibus scilicet et futuris, qualiter nos... (Sta. C. Serós, 992, p. 10) Notum facio et notum sit omnibus hominibus ... (S. V. Ov., 1045, p. 85) Notum sit omnibus ecclesie filiis presentibus scilicet ac futuris quod ego (C. H., 1093, p. 74)

Notum sit omnibus hominibus hoc audientibus quod ego (C. H., 1170, p. 267)

Notum sit presentibus et futuris quod (S. S. Oña, 1187, p. 326)

8 Los documentos altomedievales utilizados pertenecen a las siguientes colecciones: Alamo (1950), Floriano Llorente (1968), Menéndez Pidal (1966), Ser Quijano (1981), Durán Gudiol (1965-1969), Fernández Conde, Torrente Fernández, Noval Menéndez (1978), Ubieto Arteta (1960a; 1960b; 1962-1963; 1966; 1976). Dichos documentos serán citados por el nombre de la población o monasterio, de acuerdo con las siguientes abreviaturas: SC Serós: Santa Cruz de la Serós; S. V. Ov. : San Vicente de Oviedo; C. H. : Catedral de Huesca; S. S. Oña: San Salvador de Oña; S. M. : San Millán de la Cogolla; C. L. : Catedral de León; S. P. Ov. : San Pelayo de Oviedo; S. J. Peña: San Juan de la Peña; Sires.: Siresa. Asimismo indicamos el año de redacción del documento y la página en que aparece en el correspondiente cartulario. 
Notum sit cunctis presentes et futuris quod (C. H., 1187, p. 413)

Notum sit omnibus hominibus presentibus ac venturis (C. H., 1190, p. 436)

Cognitum sit quod ego (C. H., 1190, p. 440)

Manifestum (sit) presentibus et futuris hominibus quod (S. S. Oña, 1144, p. 227)

En los cartularios consultados observamos que el uso de notum sit aumenta cuando decrece el de placuit nobis atque conuenit (y variantes). Este cambio parece justificado por el Prof. García Gallo: «... Los documentos privados adquieren ahora (fines del s. XI y en el s. XII) pleno valor cuando se realizan con cierta publicidad y son confirmados por los presentes. Esta publicidad que da fuerza al documento, trata de conseguirse en el s. XII, abandonando plenamente la fórmula epistolar usual, en que el otorgante se dirige a la otra parte, sustituyéndola con una dirección general al comienzo: 'Notum sit omnibus tan presentis quam futuris ...' 0 en forma romance: 'Sepan quantos esta carta vieren u oyeren'...» (García Gallo, 1977:170).

DO ET OTORGO se representa por otros pares de verbos ${ }^{9}$

Offerimus et donamus ac confirmamus (S. M., 947, p. 61)

Dono adque concedo (C. L., 961, p. 232)

Damus et concedimus (SS Oña, 1011, p. 35 y S. V. Ov., 1083, p. 164)

Do et offero (S. P. Ov., 1145, p. 39)

Do et concedo (S. P. Ov., 1145, p. 41)

Damus atque concedimus (S. S. Oña, 1014, p. 39)

Dare atque donare (S. P. Ov., 1071, p. 26)

Do et dono (S. V. Ov., 1096, p. 197)

Damos y otorgamos aparece ya en documentos mixtos (latín/romance) a partir de 1200 según las fuentes documentales que hemos consultado ${ }^{10}$. Sin embargo, se encuentra con anterioridad qui auctorigarunt et derunt alia hereditate ... en 1028 (S. V. Ov., 1028, p. 71).

Por otra parte, hallamos el antecedente de VENDO ET OTORGO en que nos vindicaremus uel autorigare non ualunerimus (C. L., 965, p. 245).

Estas lexías complejas aparecen algunas veces acompañadas de otra tal como DE BUENA VOLUNTAD ET DE BUEN CORAÇON, que no indica sino una característica de la donación:

Grato animo et prona voluntate (C. L., 864, p. 151)

Grato animo et prona voluntate (C. L., 915, p. 171)

9 Codoñer (1972). En él trata de la permanencia o sustitución de determinados verbos en algunas fórmulas juridicas.

10 Encontramos damos e otorgamos en 1200 en Palazuelos de la Sierra, Burgos, y en 1211 en Burgos. Vid. Menéndez Pidal (1966). 
Propria et expontanea nostra uoluntate ... (C. L., 931, p. 182)

Grato animo et prona voluntatem (C. L., 950, p. 215)

Caro animo et bone voluntate (C. L., 953, p. 218)

Uono animo et expontanias meas uoluntates (C. L., 966, p. 246)

Bono animo et spontanea voluntate (S. J. Peña, 1016 y 1027)

En la descripción de las propiedades observamos una enumeración sucesiva de objetos, personas y caracteristicas, que también hallan su antecedente en latin:

«El nuestro castillo que es llamado de Paracollos con la villa et con sus terminos, con entradas et con salidas, et con aldeas pobradas et por pobrar, et con uinnas et con ortos et con moros et con ganados et con rios et con fontes et con pascos et con montes et con açenias et con molinos, ... 》 (doc. II, D)

«... quem abemus in locum predictum in Porma, ad Ofilones: corte, terras, pumares, ortales, pratus, sotos, linn (ares, c)esum uel regresum ubi potueris inuenire;...» (C. L., 997, p. 303)

"... de omnem meam ereditatem quidquid uisa sum abere in uilla quod uocidant Torre: terras, uineas, pomares, ceresiares uel omnia fructuaria, montibus, fontibus, exitos, cesum et regresum ubique de mea ereditate ...» (C. L., 950, p. 215)

"Commo en uostra cosa, libra et quita, pora dar, uender, empennar, enagenar et camiar et pora fazer della et en ella lo que quisieredes uos et los uestros...» (doc. LVII, V)

«... siat confirmata; aueatis, adeatis atque posideatis uel uindicetis, tam uos qua eciam filiis uestri uel ereditas uestra...» (C. L., 964, p. 240)

En la enumeración de las propiedades objeto del pacto hallamos en nuestros documentos la lexía CON ENTRADAS ET CONSALIDAS y en los textos latinos consultados aparece:

cessum et regressum (S. V. Ov., 948, p. 45 y C. L., 957, p. 256)

cum introitis vel exitis (S. J. Peña, 992, p. 82)

exitibus et introitibus (S. M., 957, p. 89)

cum ingressibus et egressibus (S. J. Peña, 1016, p. 104)

Esta lexía compleja va acompañada, en ocasiones, de ET CON TODAS SUS PERTINENCIAS, cuyo antecedente seria: 
(exitibus et introitibus) cum pertinentio et omni integritate (S. M., 957, p. 89)

(dedimus villas) cum omnibus illorum pertinentiis (S. J. Peña, 992 , p. 85)

Omnibus terminiis et pertinentiis suis (S. J. Peña, 1027)

(cum ingressibus et egressibus), et cum omnibus terminiis et pertinenciis suis (S. S. Oña, 1056, p. 74)

Et cum omnibus suis pertinentiis (S. S. Oña, 1096, p. 139)

Como antecedente de LIBRE ET QUITO observamos la presencia de:

Liberam in Dei Nomine abeant potestamen (S. J. Peña, h. 850, p. 26)

Liberam in Dei Nomine abeatis potestatem (S. V. Ov., 905, p. 34 y 949; p.

46)

Liberam in Dei Nomine aueas potestatem (S. V. Ov., 1046, p. 89)

Et habeatis illud liberum et quietum (S. P. Ov., 1145, p. 44)

Liberam ac sinceram habeant potestatem (Jaca, 971, p. 30)

Liberam in Dei Nomine abeant potestatem (S. J. Peña, h. 850, p. 27)

Liberam in Dei Nomine habeatis potestatem (C. L., 870, p. 153)

Liberam in Dei Nomine abeas potestatem (C. L., 892, p. 157)

MUEBLE ET RAYZ, que en otros documentos murcianos de la época aparece como bienes muebles et inmuebles, halla su precedente en

Tan inmouile quam etiam et non mouile (C. L., 870)

Tam mobilem quam eziam et inmobilem (C. L., 967)

mouile uel inmouile (C. L., 975, p. 256)

Cum omnia mea rem mobilem et inmobilem (S. M., 947, p. 63)

Tan mobile quam inmobile (S. M., 964, p. 99)

Otra de las lexías complejas enumeradas, SEA FIRME ET ESTABLE, la encontramos como

Et in antea ista carta bendictiones firmis et stabilis permaneat (Sires., 942, p. 27)

Et hunc factum nostrum sit stabile per secula cuncta amen (S. V. Ov., 1042, p. 81)

Et hec carta ... in perpetuum firmum et stabile permaneat (C. H., 1081, p. 60)

Firmun maneat et stabile perseverent (S. S. Oña, 1057, p. 75)

Una de las características de la legalización de la carta se indica en latín por medio de

Divisa per A. B. C. (varios documentos)

Asimismo encontramos antecedentes en la enumeración de testigos y en la data del documento. Entre ellos citamos los siguientes:

TESTIGOS DESTO SON

Hii sunt testes qui fuerunt presentes (S. J. Peña, h. 850, p. 28)

Et sunt testes ... (S. J. Peña, 987, p. 74) 
Qui preses fuerunt (C. L., 980)

Et hij Junt teftes (Burgos, 1100)

Et coram testibus tradimus (C. L., 967)

Qui ic preses fuerunt (C. L., 977)

LA MANDE FAZER POR mandado del maestre está representada por

Qui istam cartam mandauimus titulare et firmare (varios docs.)

QUI ESTA CARTA ESCRUI

In hanc scriptura manu mea feci (S. M., 807, p. 13)

Et ego ... scriba ... feci hanc cartam (C. H., 1066, p. 51)

Manus mea feci (C. L., 958)

FECHA EN O FECHA LA CARTA EN

Facta kartula donationis (C. L., 892)

Facta carta (Burgos, 1100)

El funcionamiento de los "escriptorios" y notarías medievales, así como la transmisión de fórmulas por los notarios, han sido temas estudiados por diversos autores ". Creemos que a través de estas páginas se puede apreciar cómo la macroestructura de las cartas medievales es esencialmente repetitiva, no sólo a lo largo de los años, sino también a lo largo de los siglos y cómo las fórmulas jurídicas que el uso ha constituido en lexías complejas hallan todas su antecedente en latín, presentando una idéntica estructura. Excepto la fórmula de llamamiento «Sepan ...", que es más tardía por las razones aducidas, las restantes comienzan a aparecer tempranamente: $h$. 850 los primeros testimonios; considerando que nuestro análisis no ha sido exhaustivo, probablemente algunas de ellas ya estarian presentes años atrás.

\section{Referencias bibliográficas}

Alamo, J. del (1950), Colección Diplomática de San Salvador de Oña, (822-1284), C.S.I.C., Madrid, 2 vols.

Codoñer, C. (1972), «Léxico de las fórmulas de donación en documentos del siglo X", en Emerita, XL, pp. 143-149.

Durán Gudiol, A. (1965 y 1969), Colección diplomática de la Catedral de Huesca. C.S.I.C._- Instituto de Estudios Pirenaicos. Zaragoza, 2 vols.

Fernández Conde, F. J., Torrente Fernández, l; Noval Menéndez, G. de la, (1978), El Monasterio de San Pelayo de Oviedo. Monasterio de San Pelayo. 2 vols.

11 Entre otros señalaremos los estudios de García Gallo (1977) y Peset - Gutiérrez Cuadrado (1981: $77-110)$. 
Floriano Llorente, P. (1968), Colección Diplomática del Monasterio de San Vicente de Oviedo. Instituto de Estudios Asturianos, CSIC, Oviedo.

García Gallo, A. (1977), «Los documentos y los formularios jurídicos en España hasta el siglo Xll». Conferencia. Academia Matritense del Notariado. 24 de noviembre de 1977, A.H.D.E., pp. 116-177.

Lalinde Abadía, J. (1970), Iniciación Histórica al Derecho Español. Ed. Ariel. Barcelona.

Menéndez Pidal, R. (1966), Documentos Lingüisticos de España (Reino de Castilla), I, Anejo de la R.F.E., Madrid. Reimpresión.

Metzeltin, M. (1982), “Segmentation sémantique d'un acte de vente médiéval» en Cahiers de Linguistique Hispanique Médiévale, 7-bis, pp. 141-155.

Pascual Martínez, L. (1982), «Los oficios y la Cancillería en el señorío de don Juan Manuel» en Don Juan Manuel. VII Centenario, Murcia, pp. 259-285.

Peset, M. y Gutiérrez Cuadrado, J. (1981): Clérigos y juristas en la Baja Edad Media castellano-leonesa. Anexo II, vol. Iil de Senara, Colexio Universitario de Vigo.

Pottier, B. (1974), Linguistique Génerale. Ed. Klincsieck. París.

Roudil, J. (1974), «Contribution au dictionnaire onomasiologique de l'espagnol médiéval» en Cahiers de Lexicologie, vol. 25, II, pp. 3-30.

- (1980), «Esquisse d'une typologie de la langue juridique des chartes».

Separata. s. I. 1061-1072.

- (1981), "Du traitement automatique des textes espagnols du Moyen Age à l'analyse sémantique: Une voie plantée d'importants jalons" en Logos Semantikos, vol. III, Madrid, Gredos, pp. 247-263.

Ser Quijano, G. del (1981), Documentación de la Catedral de León, Salamanca, Universidad de Salamanca.

Torres Fontes, J. (1969), Colección de documentos para la historia del Reino de Murcia, Vol. II, Documentos del siglo XIII, Murcia.

Ubieto Arteta, A. (1960 a), Cartulario de Siresa, Valencia.

- (1960 b), Cartulario de Albelda, Valencia.

- (1962-1963): Cartulario de San Juan de la Peña, 2 vols. Valencia.

- (1966), Cartulario de Santa Cruz de la Serós, Valencia.

- (1976), Cartulario de San Millán de la Cogolla (759-1076), Valencia. 